\title{
A Runway Incursion Detection Approach Based on Multiple Protected Area and Flight Status Machine for A-SMGCS
}

\author{
Jing $\mathrm{Li}^{1, \mathrm{a}}$, Guo Qiang Wang ${ }^{1}$, Pan $\mathrm{Zhu}^{1}$, Xin $\mathrm{Lu}^{2}$ and Jian $\mathrm{Su}^{2}$ \\ ${ }^{1} R \& D$ Centre, the Second Research Institute of Civil Aviation Administration of China (CAAC), Chengdu, China \\ ${ }^{2}$ Guilin Air Traffic Management Station of Civil Aviation of China, Guilin, Guangxi Zhuang Autonomous Region, China
}

\begin{abstract}
A-SMGCS is a modular system defined in the ICAO (International Civil Aviation Organization) Manual on Advanced Surface Movement Guidance and Control System (A-SMGCS) .One of A-SMGCS goals is to provide enhanced safety and protection of the runway. This paper presents a novel runway incursion detection approach for A-SMGCS, in which a multiple protected area is proposed to decrease complexity of pre-treatment for incursion judgment, and a flight status machine is designed to specify the transitions of one flight from one target status to another. Additionally, an HMI (Human Machine Interface) independently developed by the Second Research Institute of CAAC (Civil Aviation Administration of China) was designed in order to validate the runway incursion detection approach, the result shows that the algorithm has the potential to significantly improve runway safety by early detection and alerting of runway incursions.

Keywords: runway incursion; multiple protected area; flight status machine; A-SMGCS; ICAO
\end{abstract}

\section{Introduction}

With the increasing demand for air transport all over the world, pressure is growing for airport systems to safely handle greater capacity in all weather conditions, improve traffic distribution and management, maximize use of existing infrastructure and so on [1].The number of accidents and incidents during airport surface movements, including runway incursions, is increasing. The hazards and risks associated with runway operations need to be managed in order to prevent runway incursions that may lead to accidents. Runway incursions have sometimes led to serious accidents with significant loss of life. Although they are not a new problem, with increasing air traffic, runway incursions have been on the rise. To improve the situation with respect to runway incursions, research on Runway Incursion Prevention System (RIPS) by NASA(National Aeronautics and Space Administration) form 2002 [2] and Manual on the Prevention of Runway Incursions by ICAO from $2006[3,4]$ have been established. However, due to specific characteristics of different runways on different airports, it is too difficult to set up a model with the potential to be used at airports worldwide.

\section{ICAO definition for runway incursion in A-SMGCS}

ICAO Doc 9830 defines A-SMGCS as follows: Advanced surface movement guidance and control

\footnotetext{
a Corresponding author: casri148@163.com
}

system (A-SMGCS). A-SMGCS providing routing, guidance and surveillance for the control of aircraft and vehicles in order to maintain the declared surface movement rate under all weather conditions within the aerodrome visibility operational level while maintaining the required level of safety. A-SMGCS provides for enhanced safety and protection of the runway as one means for avoiding runway incursions [5].As described in the ICAO Doc 9830, A-SMGCS consists of four basic functions:Surveillance,Control,Planning/Routing,Guidance.Implementation of A-SMGCS defines 4 levels, the second level provides for an alerting function to Air Traffic Controllers (ATC controllers) in case of intrusion of aircraft or vehicles in a pre-defined protected area around the runway system. The third level involves the detection of all conflicts on the movement area as well as improved guidance and planning for use by controllers.

Runway Incursion defined in A-SMGCS is as follows: Any occurrence at an aerodrome involving the incorrect presence of an aircraft, vehicle or person on the protected area of a surface designated for the landing and take-off of aircraft [5].

\section{Runway incursion detection approach}

\subsection{Runway incursion area}

A major concept of the runway incursion detection algorithm is the use of incursion areas, which will help to determine the position relation of target and runway. For 
each runway, the algorithm will investigate conflicts if a track is in one of the incursion areas, depending on the type of conflict.

A runway incursion area is a software-derived threedimensional virtual area. There are multiple incursion areas for one runway. To decrease complexity about pre- treatment of incursion judgment and take into account the error of surveillance data, a multiple protected area is proposed, which consists of approach area, line-up area, runway area and departed area. Figure 1 shows the layout of the multiple protected area.

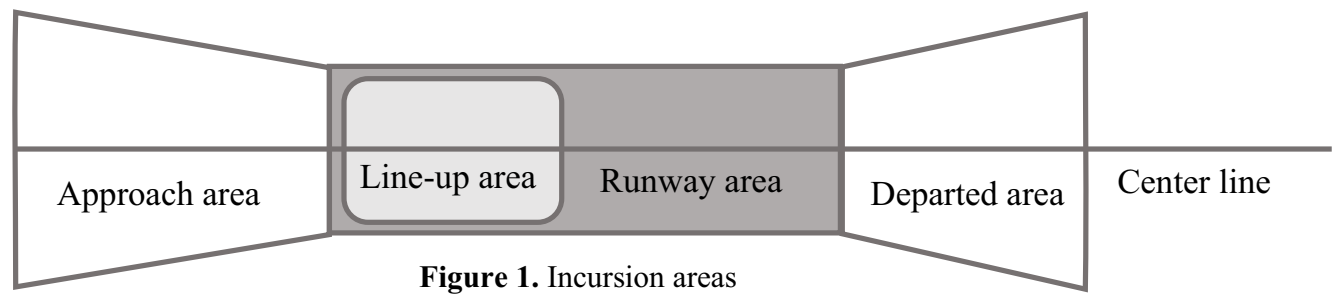

\subsection{Conflict alert}

Every aerodrome has site-specific parameters and situations to be addressed. The following list provides some of the possible conflict alert scenarios that should be detectable on the runway by the A-SMGCS:

- Aircraft arriving to, or departing aircraft on, a closed runway;

- Arriving or departing aircraft with traffic on the runway (including aircraft beyond the runwayholding positions);

- Arriving or departing aircraft with moving traffic to or on a converging or intersecting runway;

- Arriving or departing aircraft with opposite direction arrival to the runway;

- Arriving or departing aircraft with traffic crossing the runway;

- Arriving or departing aircraft with taxiing traffic approaching the runway (predicted to cross the runway-holding position);

- Arriving aircraft exiting runway at high speed with converging taxiway traffic;

- Arriving aircraft with traffic in the sensitive area(when protected);

- Aircraft exiting the runway at unintended or nonapproved locations;

- Unauthorized traffic approaching the runway;

- Unidentified traffic approaching the runway.

\subsection{Flight target status and flight status machine}

Because there are so many possible conflict scenarios, every flight target is classified as being one and only one target status to simplify the detection of complex conflict situations. Therefore, a flight status machine is designed, in which nine flight statuses or flight phases are defined for the traffic in the incursion area:

- Approaching status: track is airborne and inside approach of used runway;

- Landing status: track is runway aligned and is airborne and on final approach;

- Landed status: track is on runway;

- Taxiing status: track is on runway and is not airborne and speed is below a certain speed, or not moving;

- Line-up status: track is on runway and is in line-up area and speed is below a certain speed, or not moving;

- Rolling status: track is not airborne and ground takeoff roll is in progress;

- Departing status: track is inside runway area and speed is above a certain speed;

- Departed status: track is airborne or speed is above a certain speed;

- Unknown status: unknown intent.

A flight status machine specifies the transitions from one target status to another, figure 2 illustrates the typical transitions between statuses for arrival and departure. 


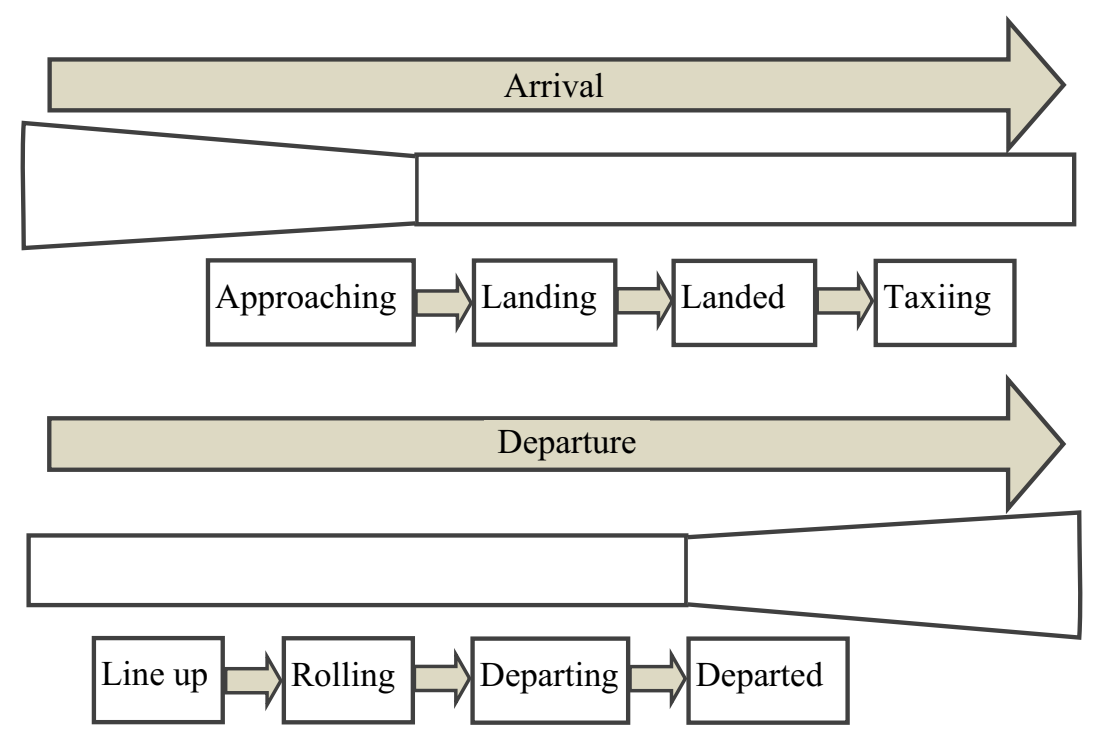

Figure 2. Typical target status transitions of one flight

There are two advantages to the flight status machine. Firstly, it avoids the possibility that a target might end up in a situation for which no target status has been defined. With a flight status machine, a target will always have a defined target status. Secondly, the flight status machine incorporates hysteresis, a technique for avoiding the problem of jumping back and forth between statuses due to surveillance errors. Figure 3 shows all the possible transitions.

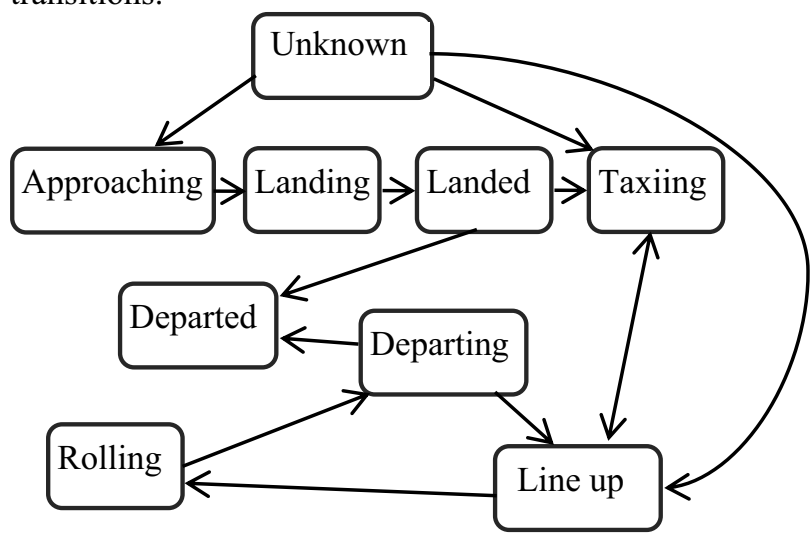

Figure 3. Possible target status transitions

\subsection{Algorithm logic and process flow}

In the present design, the alert function is event driven. The event, in this context, is the arrival of new surveillance data. When the new surveillance data is available, the alert function starts to run the detection. The alert function is based on a few steps below, and the process flow of the algorithm is shown as figure 4:

a) Step1: Decode the traffic data and initiate the target object;

b) Step2: Store flight target in a runway list if it enters one of the runway incursion areas;

c) Step3: Scan the targets in the runway list based on the pre-defined rules to detect runway incursion conflict;

d) Step4: Send alert if there is one.

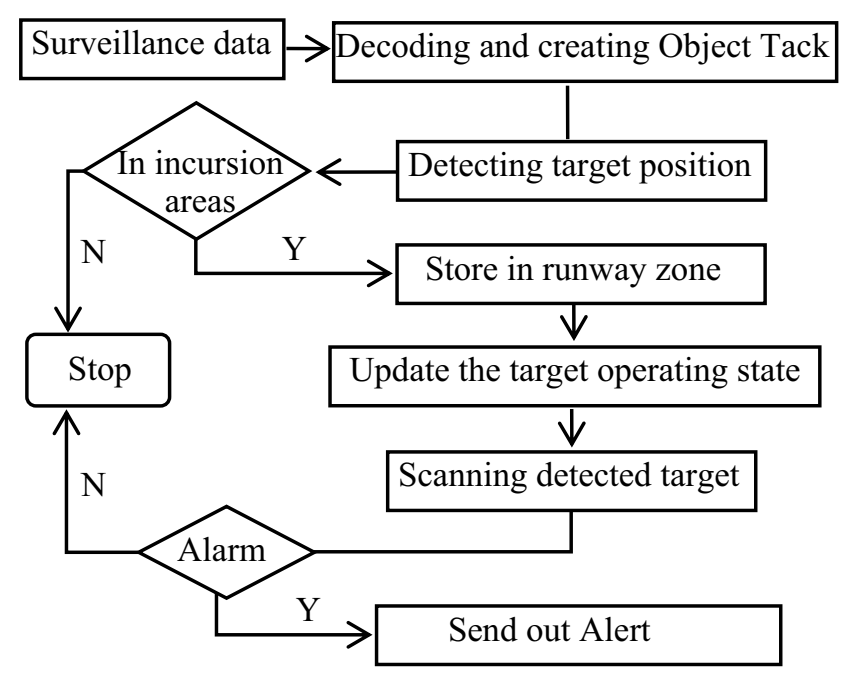

Figure 4. Process flow of the algorithm

The runway incursion detection algorithm is based on rules that define either a procedural or a physical conflict. An analysis of the nature of runway physical and procedural conflicts suggests that the conflict detection is based on four basic rules:

- Whether a movement has entered the one of the defined runway incursion areas;

- The flight target intent(target status of the target pair fits the conflict scenarios);

- Whether the two targets involved in the conflict are approaching each other;

- Whether the separation of targets is acceptable.

\section{Simulation and validation of runway incursion detection approach}

An HMI has been designed based on the runway incursion detection approach. The algorithm has been tested at Chengdu Shuangliu International Airport by real-time surveillance and simulation system independently developed by the Second Research 
Institute of CAAC, real-time data of aircraft come from Automatic Dependent Surveillance-Broadcast(ADS-B) independently developed by the Second Research Institute of CAAC as well. ATC controllers are alerted through a pop up window and labels of the aircraft concerned displayed in yellow or red, which correspond to different type of conflicts. Controllers can acknowledge conflicts by clicking on the label so that these are displayed in their normal color again. The pop up window remains active as long as the conflict is valid. In figure 5 below, a detail of the HMI is shown, which shows an aircraft has just landed at runway 02L, another one is lining up behind it for a take-off, as soon as the departing aircraft starts accelerating, a runway incursion alert is given. A large number of different incursion scenarios were designed by the real-time surveillance and simulation system according to ATC controllers' experience. Compared with the experiment on real data, the algorithm could detect common runway incursion timely and correctly. Analysis of recorded data shows that the primary performance indicators have reached the requirements on conflict detection form ICAO Doc 9830, such as:

- PADS(Probability of Detection of an Alert Situation) $>99 \%$

- PFA(Probability of False Alert $)<10^{-3}$

- $\operatorname{ART}($ Alert Response Time $)<0.5 \mathrm{~s}$

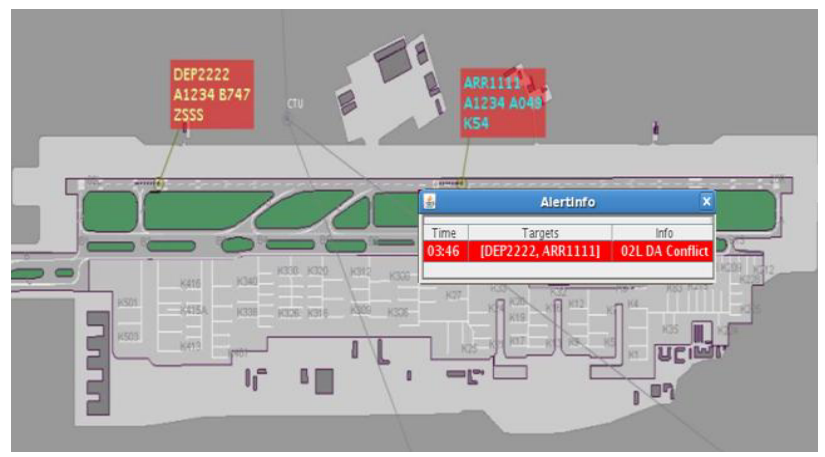

Figure 5. HMI for runway incursion detection approach

\section{Conclusion}

The runway incursion detection approach has been constructed in this article, and Chengdu Shuangliu International Airport has been chosen for simulation and validation, combing the local operational procedure, aerodrome layout, ADS-B surveillance data. Performance results and analyses from Shuangliu International Airport flight test verify that the algorithm has the potential to significantly improve runway safety by early detection and alerting of runway incursions. Although algorithm performance was highly successful, the system was not tested under all possible incursion scenarios, or in all weather and visibility conditions. More work is needed to test and fine tune the system under all conditions. Future research and development will focus on enhancement of the runway incursion detection approach, and explore taxiway incursion detection and apron incursion detection algorithm.

\section{Acknowledgement}

This paper is supported by the Technology and Innovation Fund Program of CAAC(MHRD20140218).

\section{References}

1. Qingchuan Feng. Aerodrome Control,Southwest Jiaotong University Press,2004:15-16.

2. David F. Green. Runway Safety Monitor Algorithm for Runway Incursion Detection and Alerting, NASA (2002) .

3. International Civil Aviation Organization. Manual on the Prevention of Runway Incursions, Doc9870. International Civil Aviation Organization (2006) .

4. International Civil Aviation Organization. Annex 10 to the Convention on International Civil Aviation(Volume I-Radio Navigation Aids). 6th ed. International Civil Aviation Organization(2006).

5. ICAO Doc 9830: Advanced Surface Movement Guidance and Control Systems (A-SMGCS) Manual(2004). 